\title{
Prognostic and clinicopathological significance of SNHG20 in human cancers: a meta-analysis
}

Hanlong Zhu ${ }^{\dagger}$, Si Zhao ${ }^{\dagger}$, Ruonan Jiao, Huishan Wang, Ruiyi Tang, Xiaochao Wu, Fei Wang, Xianxiu Ge, Quanpeng Li and Lin Miao* (1)

\begin{abstract}
Background: It has been widely reported that the expression levels of SNHG20 are elevated in diverse types of cancers, indicating that SNHG20 may participate in cancer initiation and development. Besides, accumulating evidence reveals that SNHG2O overexpression is also connected with poor clinical outcomes among cancer patients. Herein, we carry out a systematic meta-analysis to further determine the prognostic and clinical significance of SNHG20 expression in various human cancers.
\end{abstract}

Methods: Qualifying publications were selected by searching for keywords in PubMed, Embase, Web of Science and Cochrane Library databases, up to September 1, 2019. Pooled hazard ratio (HR) or odds ratio (OR) with corresponding $95 \%$ confidence interval (Cl) was computed to estimate the strength of association between SNHG20 and survival of cancer patients or clinicopathology using Stata 14.0 software.

Results: In total, 15 studies encompassing 1187 patients met the inclusion criteria were ultimately enrolled for analysis. According to the meta-analysis, patients with high SNHG20 expression were markedly linked to poorer overall survival (OS) (pooled $\mathrm{HR}=2.47,95 \% \mathrm{Cl} 2.05-2.98, \mathrm{P}=0.000$ ) and disease-free survival/recurrence-free sur$\mathrm{vival} /$ progression-free survival (DFS/RFS/PFS) (pooled $H R=2.37,95 \% \mathrm{Cl} 1.60-3.51, \mathrm{P}=0.000$ ). Additionally, regarding clinicopathology of patients, enhanced SNHG20 was correlated with advanced tumour-node-metastasis (TNM) stage $(\mathrm{OR}=2.80,95 \% \mathrm{Cl} 2.00-3.93, \mathrm{P}=0.000)$, larger tumor size $(\mathrm{OR}=3.08,95 \% \mathrm{Cl} 2.11-4.51, \mathrm{P}=0.000)$, positive lymph nodes metastasis $(\mathrm{OR}=2.99,95 \% \mathrm{Cl} 2.08-4.31, \mathrm{P}=0.000)$, higher tumor stage $(\mathrm{OR}=4.51,95 \% \mathrm{Cl} 2.17-9.37, \mathrm{P}=0.000)$ and worse histological grade $(\mathrm{OR}=1.95,95 \% \mathrm{Cl} 1.44-2.63, \mathrm{P}=0.000)$, but not with gender, smoking status or distant metastasis.

Conclusions: Up-regulated SNHG20 expression is ubiquitous in different kinds of cancers. Moreover, up-regulated SNHG20 expression is capable of serving as an innovative predictive factor of inferior clinical outcomes in cancer patients. Nevertheless, higher-quality multicenter studies are required to corroborate our results.

Keywords: SNHG20, IncRNA, Cancer, Prognosis, Meta-analysis

*Correspondence: linmiao@njmu.edu.cn

${ }^{\dagger}$ Hanlong Zhu and Si Zhao contributed equally to this work

Medical Centre for Digestive Diseases, The Second Affiliated Hospital of Nanjing Medical University, Nanjing 210011, Jiangsu, People's Republic of China

\section{Background}

Cancer has become one of the common chronic diseases that seriously threatens human health and imposes an immense burden on society. The upward trend of cancer gives rise to worldwide concern, with almost 1,762,450 newly diagnosed cancer cases and

(c) The Author(s) 2020. This article is licensed under a Creative Commons Attribution 4.0 International License, which permits use, sharing, adaptation, distribution and reproduction in any medium or format, as long as you give appropriate credit to the original author(s) and the source, provide a link to the Creative Commons licence, and indicate if changes were made. The images or other third party material in this article are included in the article's Creative Commons licence, unless indicated otherwise in a credit line to the material. If material is not included in the article's Creative Commons licence and your intended use is not permitted by statutory regulation or exceeds the permitted use, you will need to obtain permission directly from the copyright holder. To view a copy of this licence, visit http://creativeco mmons.org/licenses/by/4.0/. The Creative Commons Public Domain Dedication waiver (http://creativecommons.org/publicdomain/ zero/1.0/) applies to the data made available in this article, unless otherwise stated in a credit line to the data. 
approximately 606,880 cancer-related deaths in the United States in 2019 [1]. Although the survival benefit of multidisciplinary synthetic therapy is recognized, the prognosis in patients with terminal stages of cancer remains unsatisfactory [2]. To this end, the identification of an accurate biomarker for cancer prognosis is of great clinical value, which can be applied to early diagnosis and targeted therapy for clinical practice.

More recently, with the advent of next-generation sequencing technologies, long noncoding RNAs (lncRNAs), as a new category of noncoding transcripts, have come into the spotlight [3]. By definition, lncRNAs constitute a large and heterogeneous subset of RNAs that are distinguished by a length of greater than 200 nucleotides and the absence of protein-coding capability [4]. They were once regarded as simply genomic "junk" so that they having been underappreciated for a long time [5]. Nonetheless, lncRNAs have emerged as functional molecules, which serve pivotal roles in diverse biological processes, with clear relevance to cancer [5]. Additionally, accumulating shreds of evidence unveil that lncRNAs can exert oncogenic or tumor-suppressing effects in tumorigenesis and progression [6], suggesting that lncRNAs may be candidate markers for cancer diagnosis, prognosis, and therapeutics.

The small nucleolar RNA host gene 20 (SNHG20) has arrested our attention among the lncRNAs, which stems from chromosome 17q25.2 and harbors 2183 basepairs [7]. Initially, SNHG20 was discovered in hepatocellular carcinoma (HCC) and has been proved to function as an oncogene in HCC [8]. Subsequently, a growing body of research has identified that aberrant SNHG20 expression definitely interacted with prognostic outcomes and clinicopathological characteristics in patients with many kinds of malignancies, including bladder cancer [9], osteosarcoma [10], glioma [11], colorectal cancer [12], gastric cancer [13], lung cancer [14], cervical cancer [15], esophageal carcinoma [16], oral carcinoma [17], nasopharyngeal carcinoma [18], ovarian cancer [19], and laryngeal carcinoma [20]. Consistently, overexpression of SNHG20 could promote cell-cycle, proliferation, invasion, and migration of tumor cells via different mechanisms, while up-regulated SNHG20 is an unfavorable prognostic factor [7]. It should be taken into account that most individual studies are restricted by controversial and discrete conclusions as well as small sample size. For the sake of comprehensively validating the underlying prognostic and clinicopathological role of SNHG20 in various malignancy patients, a quantitative meta-analysis is therefore undertaken.

\section{Materials and methods}

\section{Literature search strategies}

Up to September 1, 2019, potential eligible literature were systematically retrieved in four authoritative databases including PubMed, Embase, Web of Science and Cochrane Library databases to obtain pertinent articles regarding prognostic and clinicopathological features of SNHG20 among various tumors. The searched keywords in variably combinations were as following: ("small nucleolar RNA host gene 20" OR "SNHG20") AND ("cancer" OR "carcinoma" OR "tumor" OR "neoplasm") AND ("prognosis" OR "prognostic"). The reference lists of included studies were also checked to identify potential relevant papers.

\section{Inclusion and exclusion criteria}

The research involved in this meta-analysis were asked to meet the following preassigned criteria: (1) investigated the roles of SNHG20 in multiple human tumors, (2) detected the expression levels of SNHG20 in cancer tissue, (3) divided the patients into dichotomous groups according to the specific criteria for SNHG20 expression levels, (4) reported data related with the clinicopathological characteristics and prognostic information of the patients, and (5) had sufficient data for calculating the hazard ratios (HR) with corresponding 95\% confidence intervals $(\mathrm{CI})$. All these studies were not included because of the any of the following reasons: (1) stated reduplicative research, (2) offered insufficient or unavailable data, (3) were reviews, letters, case reports, editorials, expert opinions, conference reports, and animal experiments, and (4) published in a non-English language.

\section{Literature screening and data extraction}

Two investigators (Hanlong Zhu, Si Zhao) independently screened the literature following the prespecified criteria described above and extracted the data. Any conflicts were resolved through consensus with a third scholar (Ruonan Jiao). The following information was collected from each enrolled study: lead author name, publication year, region, carcinoma type, sample size (high/low), SNHG20 assessment method and the cut-off approximations for SNHG20 expression levels, the clinicopathological parameters including age, gender, smoking status, tumour-node-metastasis (TNM) stage, tumor size, lymph node metastasis, tumor stage, histological grade, and distant metastasis, together with $\mathrm{HR}$ and $95 \% \mathrm{CI}$ for overall survival (OS), disease-free survival (DFS), recurrencefree survival (RFS) and progression-free survival (PFS). If only Kaplan-Meier curves existed in some articles, HR and $95 \% \mathrm{CI}$ were determined with available data using the published method [21, 22]. 


\section{Quality evaluation}

The quality of eligible publications was calculated based on the Newcastle-Ottawa Scale (NOS) that evaluated the selection of cohorts, comparability as well as exposure or outcome and had a score ranging from 0 to 9 . Studies with higher or equal to 6 points could be considered as high quality (Table 1).

\section{Statistical analysis}

The pooled HR with corresponding 95\% CI was utilized to estimate the relationship between SNHG20 expression and patients' prognosis. While the effect of SNHG20 expression on clinicopathological features was described as the combined odds ratio (OR) and matching 95\% CI. Cochran's $\mathrm{Q}$ and $\mathrm{I}^{2}$ tests were applied for checking the heterogeneity of the results. A $\mathrm{P}$ value $<0.1$ suggested having statistical significance, whereas $\mathrm{I}^{2}$ values $>50 \%$ indicated the existence of significant heterogeneity. When there was homogeneous data, the fixed-effect framework was adopted, otherwise, the random-effect model was employed. Besides, probable publication bias was quantified with conducting Begg's test and Egger's test, respectively. Sensitivity analysis was also done to investigate the stability of the accumulated results. All analyses were carried out using Stata 14.0 software. P value $<0.05$ was regarded as being statistically significant.

\section{Results}

\section{Data selection and characteristics}

According to the search strategy, 89 relevant records were initially retrieved from four electronic databases. Three publications were enrolled by manually searching the reference lists. After ruling out the duplicates, 45 studies were left for further assessment. Next, 17 papers were directly removed by carefully scanning titles and abstracts. For the remaining 28 articles, 13 articles were excluded owing to lack of sufficient data. Ultimately, 15 studies showing agreement with the inclusion criteria were selected for entering in the meta-analysis (Fig. 1).

The attributes of the research studies involved in the present analysis are summarized in Table 1 . These studies containing 1187 cancer patients had an accrual period between 2016 and 2019 and sample sizes varying from 32 to 144 (mean, 79). Each and every research study was performed in China; two studies referred to hepatocellular carcinoma $[8,23]$, two studies involved osteosarcoma $[10,24]$, two studies touched upon glioma [11, 25], and the remaining nine studies related to colorectal cancer [12], gastric cancer [13], lung cancer [14], cervical cancer [15], esophageal carcinoma [16], oral carcinoma [17], nasopharyngeal carcinoma [18], ovarian cancer [19], and laryngeal carcinoma [20]. The whole subjects registered were separated into high and low SNHG20 group on the basis of the SNHG20 measurement results. Moreover, 14 studies used quantitative reverse transcription polymerase chain reaction (qRT-PCR) for the detection of SNHG20 expression in tumor tissues while only one study chose in situ hybridization (ISH). Most articles preferred to exploit the median value as the Cut-off value for high or low SNHG20 expression. Regarding survival outcomes, all of the cohorts reported patients' OS, of which four cohorts simultaneously presented DFS/RFS/PFS. Among the studies, $\mathrm{HR}$ and $95 \% \mathrm{CI}$ were provided in five original articles and indirectly reckoned from survival curves in the rest of ten papers. Overall, all these qualified studies were recognized to be of high quality in this meta-analysis.

\section{Association between SNHG20 expression and survival of cancer patients SNHG20 expression and OS}

A total of fifteen studies comprising 1187 patients focused on assessing the effect of SNHG20 overexpression on OS in various kinds of cancer. As illustrated in Fig. 2a, a fixed-effect framework was applied because of the lack of significant heterogeneity among these studies $\left(\mathrm{I}^{2}=0.0 \%, \mathrm{P}=0.718\right)$. The pooled $\mathrm{HR}$ suggested that the high SNHG20 expression group showed a statistically obvious decline in OS (pooled $\mathrm{HR}=2.47,95 \% \mathrm{CI}$ 2.05-2.98, $\mathrm{P}=0.000$ ). In addition, subgroup analyses were performed regarding cancer types, sample sizes and data extraction methods to further analyze the predictive value of SNHG20 (Fig. 2b-d, Table 2). In the stratified analysis by type of cancers, promoted SNHG20 expression status was closely related to worse OS of the patients with respiratory system cancers (pooled $\mathrm{HR}=3.78,95 \%$ CI 1.18-12.09, $\mathrm{P}=0.025$, fixed-effect), gliomas (pooled $\mathrm{HR}=3.27,95 \%$ CI $1.84-5.82, \mathrm{P}=0.000$, fixed-effect), digestive system cancers (pooled $\mathrm{HR}=2.91,95 \% \mathrm{CI}$ 2.16-3.92, $\mathrm{P}=0.000$, fixed-effect), head and neck cancers (pooled HR $=1.97,95 \%$ CI 1.84-5.82, $\mathrm{P}=0.000$, fixedeffect) and osteosarcomas (pooled $\mathrm{HR}=1.95,95 \% \mathrm{CI}$ 1.23-3.09, $\mathrm{P}=0.005$, fixed-effect), apart from reproductive system cancers (pooled HR $=2.16,95 \%$ CI $0.95-4.87$, $\mathrm{P}=0.065$, fixed-effect). When the studies were categorized according to sample sizes, a significant connection was observed between SNHG20 upregulation and inferior OS in large sample sizes $(>100$, pooled $H R=2.86$, 95\% CI 2.09-3.92, $\mathrm{P}=0.000$, fixed-effect), middle sample sizes $(80-100$, pooled $H R=2.64,95 \%$ CI $1.81-3.87$, $\mathrm{P}=0.000$, fixed-effect) or small sample sizes $(<80$, pooled $\mathrm{HR}=2.09$, 95\% CI 1.56-2.81, $\mathrm{P}=0.000$, fixed-effect), demonstrating that larger sample sizes might devote to more robust and accurate results. As for different data extraction methods, the subgroup analysis unveiled that 


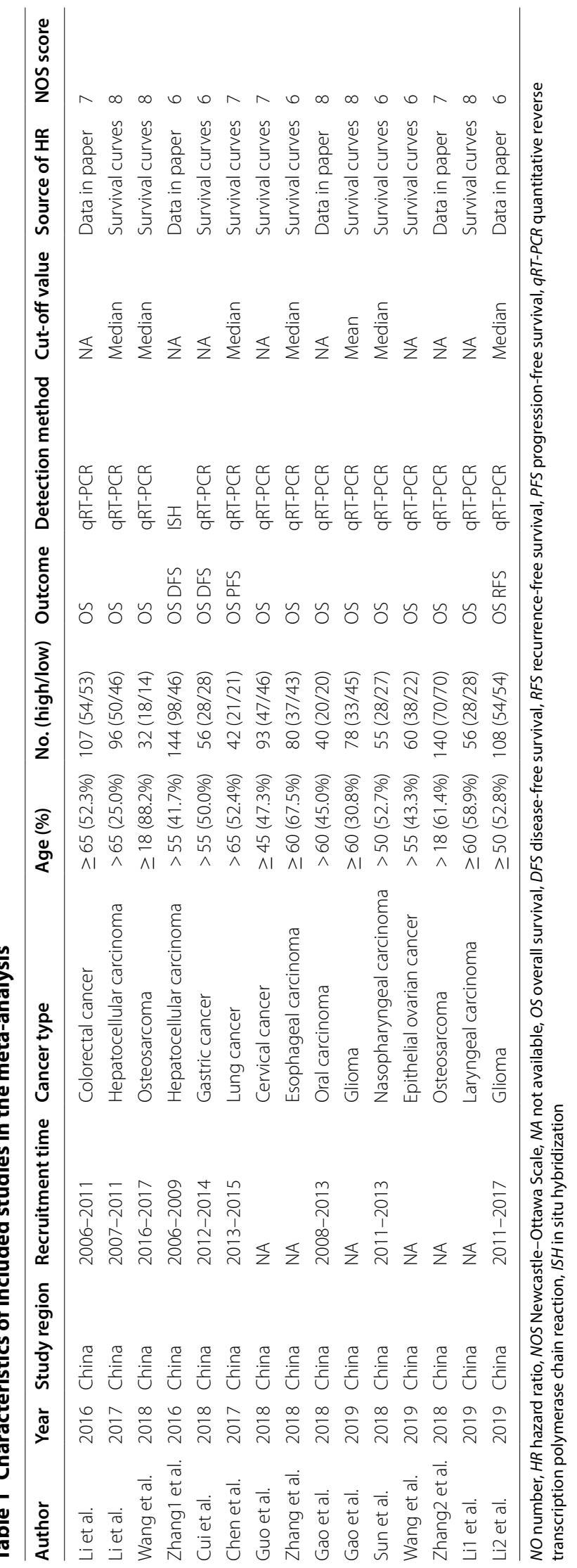




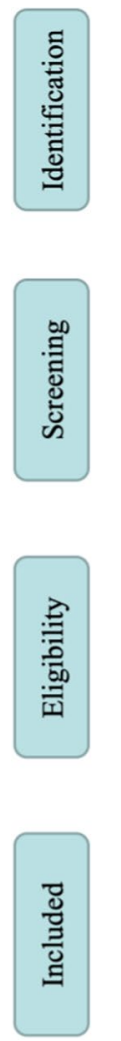

Records identified through
PubMed, Embase, Cochrane
Library and Web of Science searching $(n=89)$

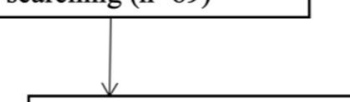

Records after duplicates removed $(n=45)$

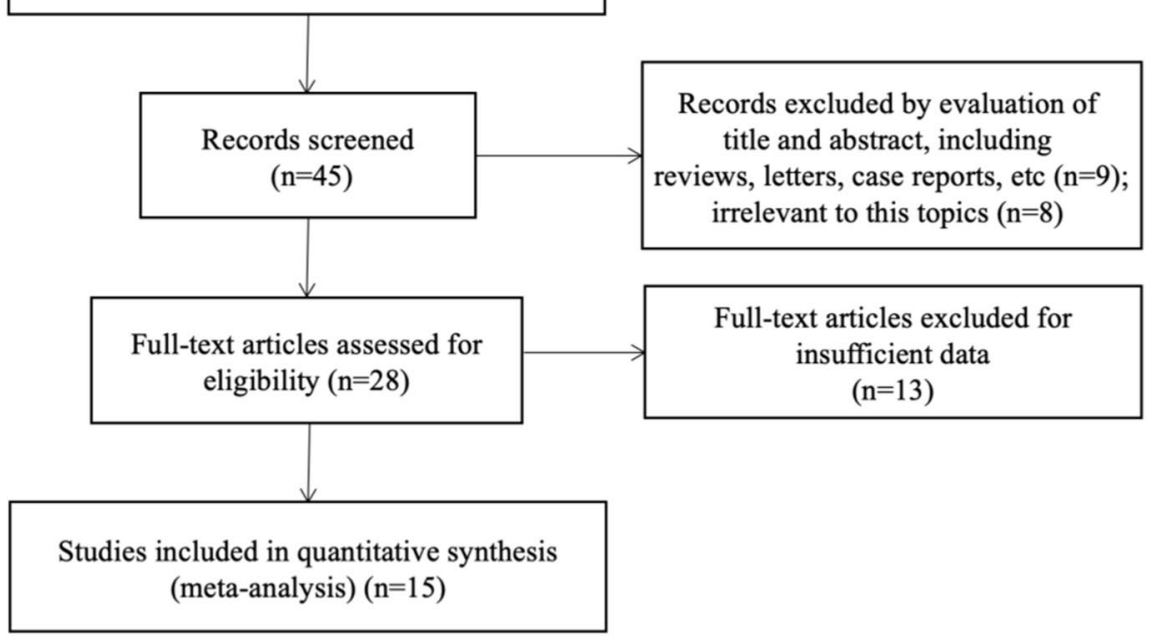

Fig. 1 Flow diagram of the process for study selection

the prognostic value of SNHG20 on the OS was not influenced by data extraction methods, that is, the HR provided in the papers (pooled $\mathrm{HR}=2.55,95 \% \mathrm{CI} 1.98-3.29$, $\mathrm{P}=0.000$, fixed-effect) or extracted from the survival curves (pooled $\mathrm{HR}=2.38,95 \%$ CI $1.80-3.14, \mathrm{P}=0.000$, fixed-effect). No severe heterogeneity was checked within the subgroups.

\section{SNHG20 expression and DFS/RFS/PFS}

Four articles consisting of 350 cases exhibited the prognostic role of SNHG20 on cancer progression or recurrence, with a pooled HR of 2.37 (95\% CI 1.60-3.51, $\mathrm{P}=0.000$, Fig. 3). Of note, enforced SNHG20 expression predicted a poor performance for DFS/RFS/PFS in the involved cancer types compared with low SNHG20 expression. No any significant heterogeneity existed across studies under a fixed-effect model $\left(\mathrm{I}^{2}=0.0 \%\right.$, $\mathrm{P}=0.974)$.

\section{Correlation between SNHG20 expression and clinical characteristics in patients with cancer TNM stage}

Reports from an aggregate of nine studies declared the correlation of SNHG20 with TNM stage in multiple tumors, with a fixed-effect model on account of limited heterogeneity $\left(\mathrm{I}^{2}=13.9 \%, \mathrm{P}=0.318\right)$. The combined analysis highlighted that patients with elevated SNHG20 expression had a tendency for more advanced TNM phase $(\mathrm{OR}=2.80,95 \%$ CI $2.00-3.93, \mathrm{P}=0.000$, Fig. $4 \mathrm{a}$, Table 3).

\section{Tumor size}

In total, six studies with 478 patients were employed to disclose a link between SNHG20 expression and tumor size. Due to insignificant heterogeneity, a fixed-effect framework was adopted $\left(\mathrm{I}^{2}=0.0 \%, \mathrm{P}=0.753\right)$. Obviously, this association demonstrated that patients with increased SNHG20 expression, were more liable to develop large tumor size $(\mathrm{OR}=3.08,95 \%$ CI $2.11-4.51$, $\mathrm{P}=0.000$, Fig. 4b, Table 3).

\section{Lymph node metastasis}

The relationship between SNHG20 expression and lymph node metastasis was evaluated in eight studies containing 534 patients. A fixed-effect model was applied to calculate the accumulated OR and its 95\% CI, when there was marginally moderate heterogeneity between studies $\left(\mathrm{I}^{2}=45.0 \%, \mathrm{P}=0.079\right)$. The aggregated results suggested 

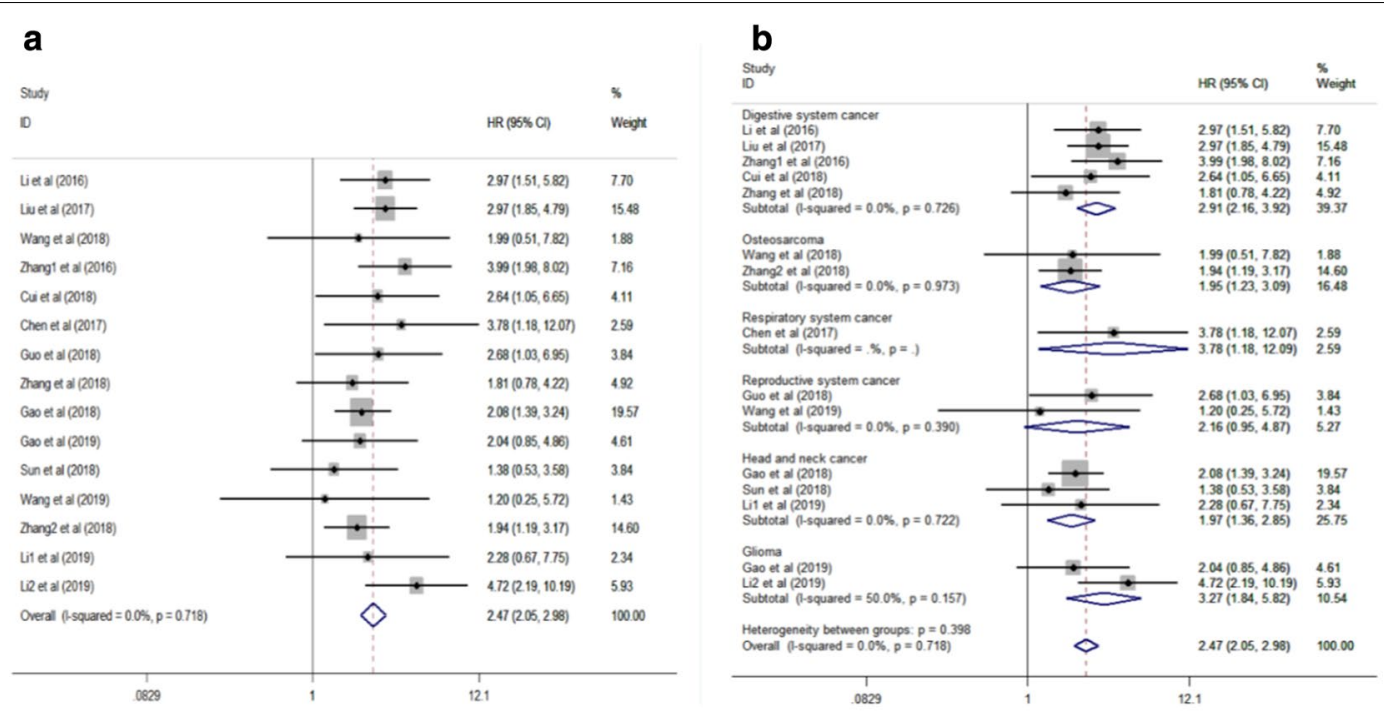

C
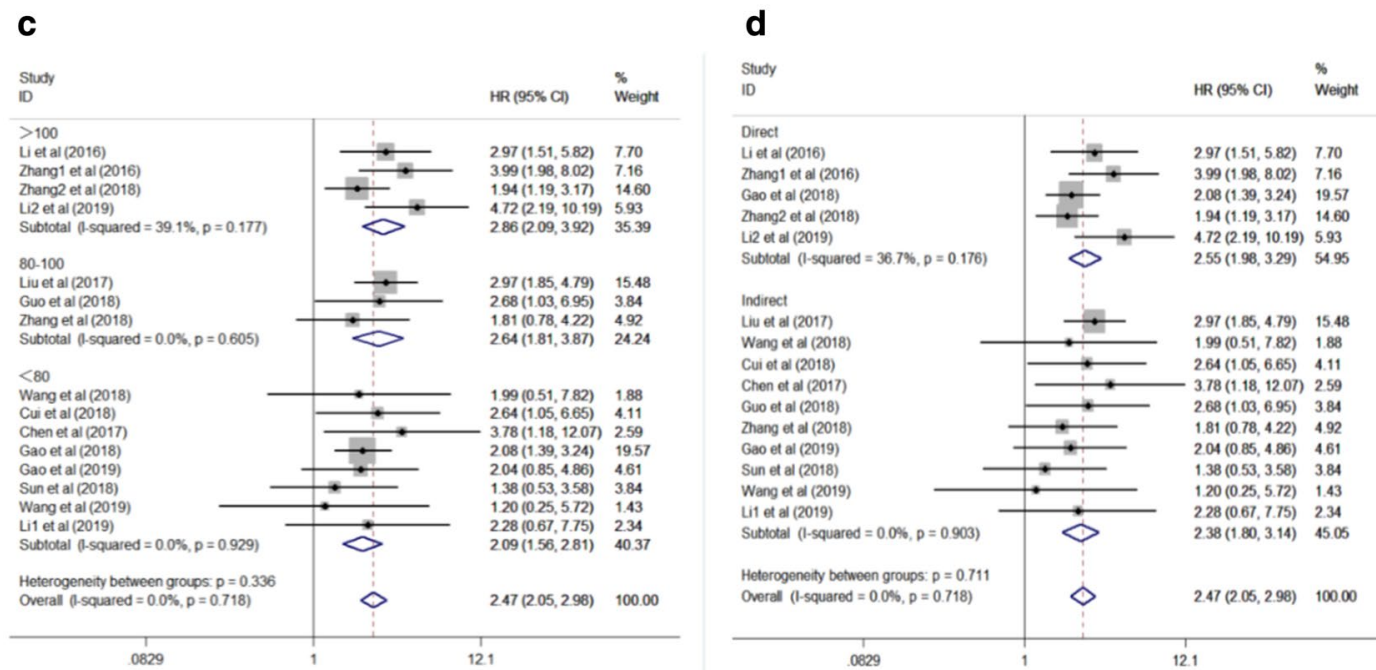

Fig. 2 Forest plots for the association between SNHG20 expression and OS. a overall; b cancer type; c sample size; d extracted method. OS overall survival, HR hazard ratio, 95\% Cl 95\% confidence interval

that patients with up-regulated SNHG20 expression preferentially metastasized to the lymph nodes $(\mathrm{OR}=2.99$, $95 \%$ CI 2.08-4.31, $\mathrm{P}=0.000$, Fig. 4c, Table 3).

\section{Tumor stage}

Three studies described the tumor stage of 203 patients in the light of different SNHG20 expression levels. No evidence of statistical heterogeneity was found; consequently, a fixed-effect framework was performed to pool the results $\left(\mathrm{I}^{2}=0.0 \%, \mathrm{P}=0.943\right)$. This showed that the patients with augmented SNHG20 expression tended towards high tumor stage $(\mathrm{OR}=4.51,95 \%$ CI $2.17-9.37$, $\mathrm{P}=0.000$, Table 3 ).

\section{Histological grade}

There were nine studies revealed a connection between SNHG20 expression and histological grade, and data of 844 patients were collected and pooled for reanalysis. A fixed-effect model was utilized for low heterogeneity detected among included studies $\left(\mathrm{I}^{2}=6.0 \%, \mathrm{P}=0.385\right)$. Statistical analyses illustrated the fact that patients with SNHG20 over-expression had a higher risk of poor histological grade $(\mathrm{OR}=1.95,95 \% \mathrm{CI} 1.44-2.63, \mathrm{P}=0.000$, Fig. 4d, Table 3).

Nevertheless, no conspicuous association was observed between SNHG20 expression and gender $(\mathrm{OR}=0.96,95 \%$ CI $0.74-1.25, \mathrm{P}=0.763$, fixed-effect, Table 3$)$, smoking status (OR $=1.16,95 \%$ CI $0.59-2.28$, 
Table 2 Overall and subgroup meta-analysis of the association between SNHG20 expression and OS

\begin{tabular}{|c|c|c|c|c|c|c|c|}
\hline \multirow[t]{2}{*}{ Subgroup } & \multirow[t]{2}{*}{ Studies/N } & \multirow[t]{2}{*}{ Patient/N } & \multirow[t]{2}{*}{ Pooled HR (95\% Cl) } & \multirow[t]{2}{*}{$P$ value } & \multicolumn{3}{|c|}{ Heterogeneity } \\
\hline & & & & & $\mathbf{1}^{2}$ & $P$ value & Model \\
\hline Overall & 15 & 1187 & $2.47(2.05,2.98)$ & 0.000 & $0.00 \%$ & 0.718 & Fixed-effect \\
\hline \multicolumn{8}{|l|}{ Cancer type } \\
\hline Digestive system cancer & 5 & 483 & $2.91(2.16,3.92)$ & 0.000 & $0.00 \%$ & 0.726 & Fixed-effect \\
\hline Osteosarcoma & 2 & 172 & $1.95(1.23,3.09)$ & 0.005 & $0.00 \%$ & 0.973 & Fixed-effect \\
\hline Respiratory system cancer & 1 & 42 & $3.78(1.18,12.09)$ & 0.025 & - & - & - \\
\hline Reproductive system cancer & 2 & 153 & $2.16(0.95,4.87)$ & 0.065 & $0.00 \%$ & 0.390 & Fixed-effect \\
\hline Head and neck cancer & 3 & 151 & $1.97(1.36,2.85)$ & 0.000 & $0.00 \%$ & 0.722 & Fixed-effect \\
\hline Glioma & 2 & 186 & $3.27(1.84,5.82)$ & 0.000 & $50.00 \%$ & 0.157 & Fixed-effect \\
\hline \multicolumn{8}{|l|}{ Sample size } \\
\hline$>100$ & 4 & 499 & $2.86(2.09,3.92)$ & 0.000 & $39.10 \%$ & 0.177 & Fixed-effect \\
\hline 80-100 & 3 & 269 & $2.64(1.81,3.87)$ & 0.000 & $0.00 \%$ & 0.605 & Fixed-effect \\
\hline$<80$ & 8 & 419 & $2.09(1.56,2.81)$ & 0.000 & $0.00 \%$ & 0.929 & Fixed-effect \\
\hline \multicolumn{8}{|l|}{ Extracted method } \\
\hline Direct & 5 & 539 & $2.55(1.98,3.29)$ & 0.000 & $36.70 \%$ & 0.176 & Fixed-effect \\
\hline Indirect & 10 & 648 & $2.38(1.80,3.14)$ & 0.000 & $0.00 \%$ & 0.903 & Fixed-effect \\
\hline
\end{tabular}

OS Overall survival, $H R$ hazard ratio, $95 \%$ Cl $95 \%$ confidence interval

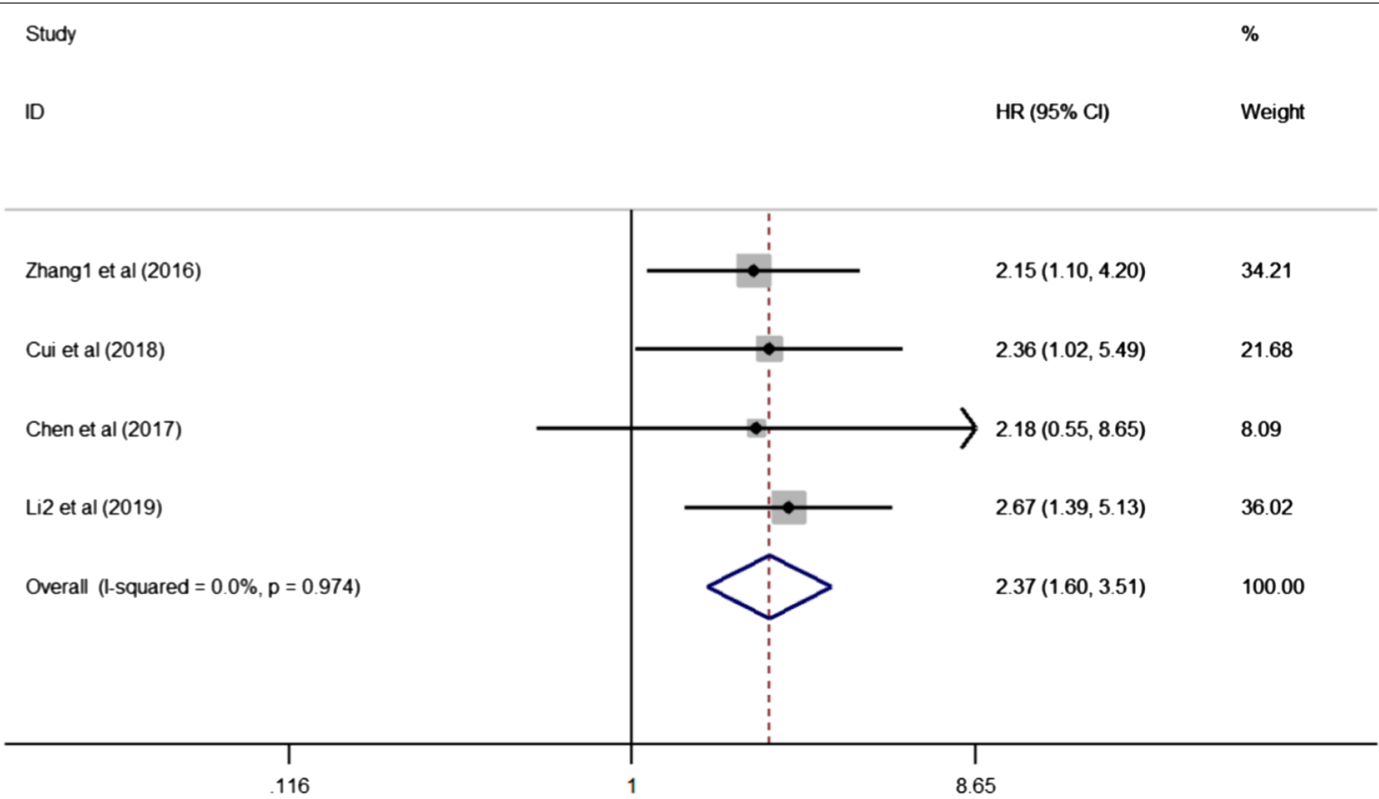

Fig. 3 Meta-analysis for the pooled HRs of DFS/RFS/PFS in patients with various cancers. DFS disease-free survival, RFS recurrence-free survival, PFS progression-free survival, HR hazard ratio, 95\% Cl 95\% confidence interval

$\mathrm{P}=0.676$, fixed-effect, Table 3) or distant metastasis $(\mathrm{OR}=1.28,95 \%$ CI $0.35-4.71, \mathrm{P}=0.706$, randomeffect, Table 3 ).

\section{Sensitivity analysis}

A sensitivity analysis was conducted by omitting individual research by turns with the aim to explore the stability of meta-analysis of SNHG20 and OS. As presented in
Fig. 5, the cumulative HR was not dramatically impacted, which further substantiated the reliability and validity of our results.

\section{Assessment of publication bias}

Publication bias was examined with respect to the survival endpoints of OS by introducing Funnel plots, Begg's and Egger's test. The symmetrical funnel plot (Fig. 6), 


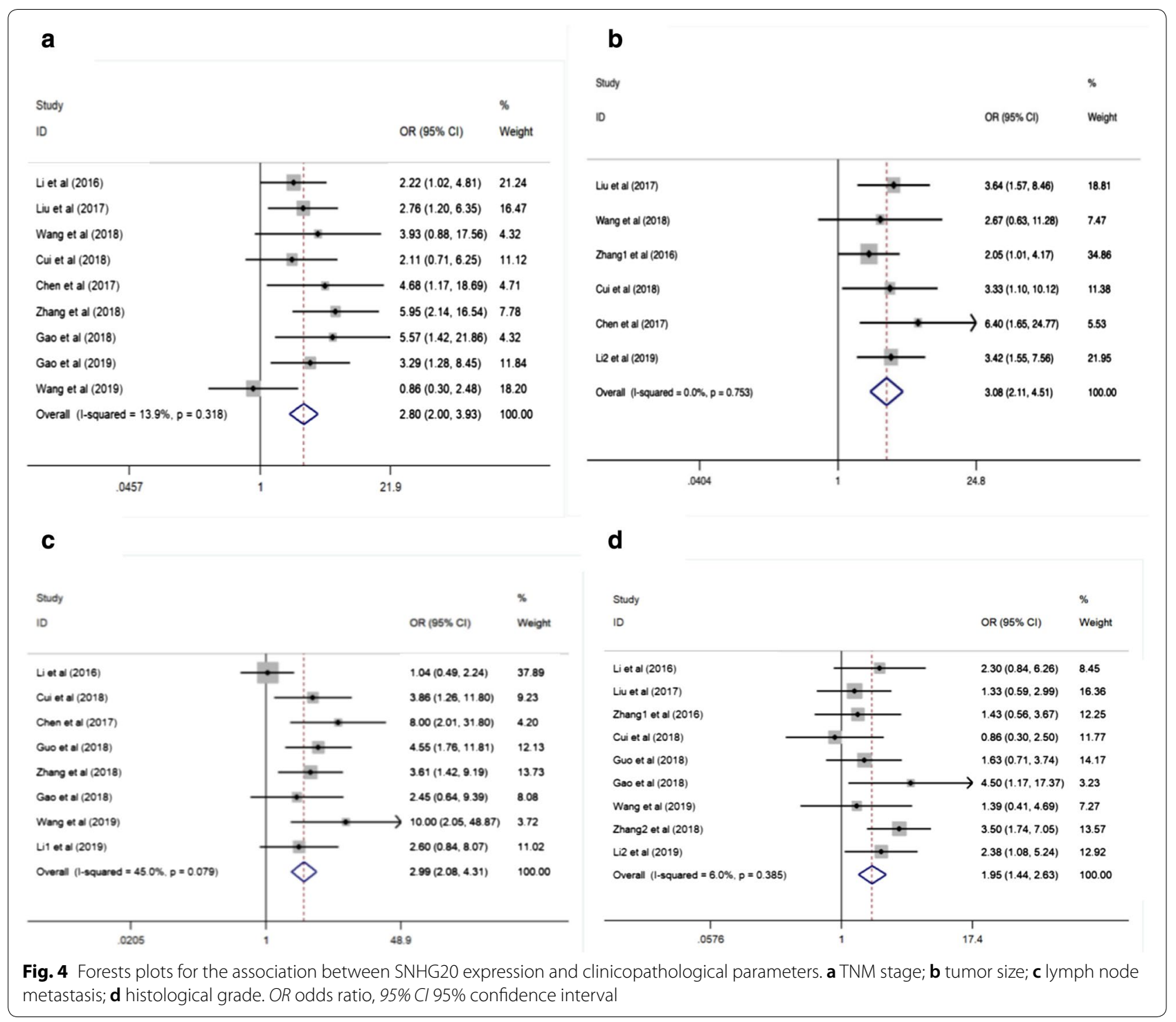

Table 3 Meta analysis results for the association of over-expressed SNHG20 with clinicopathological parameters

\begin{tabular}{|c|c|c|c|c|c|c|c|c|c|}
\hline \multirow[t]{2}{*}{ Categories } & \multirow[t]{2}{*}{ Studies (n) } & \multirow{2}{*}{$\begin{array}{l}\text { Number } \\
\text { of patients }\end{array}$} & \multirow[t]{2}{*}{ OR (95\% Cl) } & \multirow[t]{2}{*}{$P$ value } & \multicolumn{3}{|c|}{ Heterogeneity } & \multirow[t]{2}{*}{ Begg } & \multirow[t]{2}{*}{ Egger } \\
\hline & & & & & $I^{2}(\%)$ & P value & Model & & \\
\hline Gender (male vs female) & 13 & 1034 & $0.96(0.74,1.25)$ & 0.763 & 0.00 & 0.607 & Fixed-effect & 0.951 & 0.792 \\
\hline Smoking status (yes vs no) & 3 & 137 & $1.16(0.59,2.28)$ & 0.676 & 0.00 & 0.439 & Fixed-effect & - & - \\
\hline Distant metastasis (yes vs no) & 5 & 502 & $1.28(0.35,4.71)$ & 0.706 & 84.50 & 0.000 & Random-effect & - & - \\
\hline TNM stage (III/IV vs I/II) & 9 & 591 & $2.80(2.00,3.93)$ & 0.000 & 13.90 & 0.318 & Fixed-effect & 0.348 & 0.389 \\
\hline Tumor size ( $>5 \mathrm{~cm}$ vs $<5 \mathrm{~cm}$ ) & 6 & 478 & $3.08(2.11,4.51)$ & 0.000 & 0.00 & 0.753 & Fixed-effect & - & - \\
\hline Lymph node metastasis (yes vs no) & 8 & 534 & $2.99(2.08,4.31)$ & 0.000 & 45.00 & 0.079 & Fixed-effect & - & - \\
\hline Tumor stage (T3/T4 vs T1/T2) & 3 & 203 & $4.51(2.17,9.37)$ & 0.000 & 0.00 & 0.943 & Fixed-effect & - & - \\
\hline Histological grade (poorly vs well/moderately) & 9 & 844 & $1.95(1.44,2.63)$ & 0.000 & 6.00 & 0.385 & Fixed-effect & 0.602 & 0.575 \\
\hline
\end{tabular}


Meta-analysis estimates, given named study is omitted | Lower Cl Limit

Estimate

| Upper Cl Limit

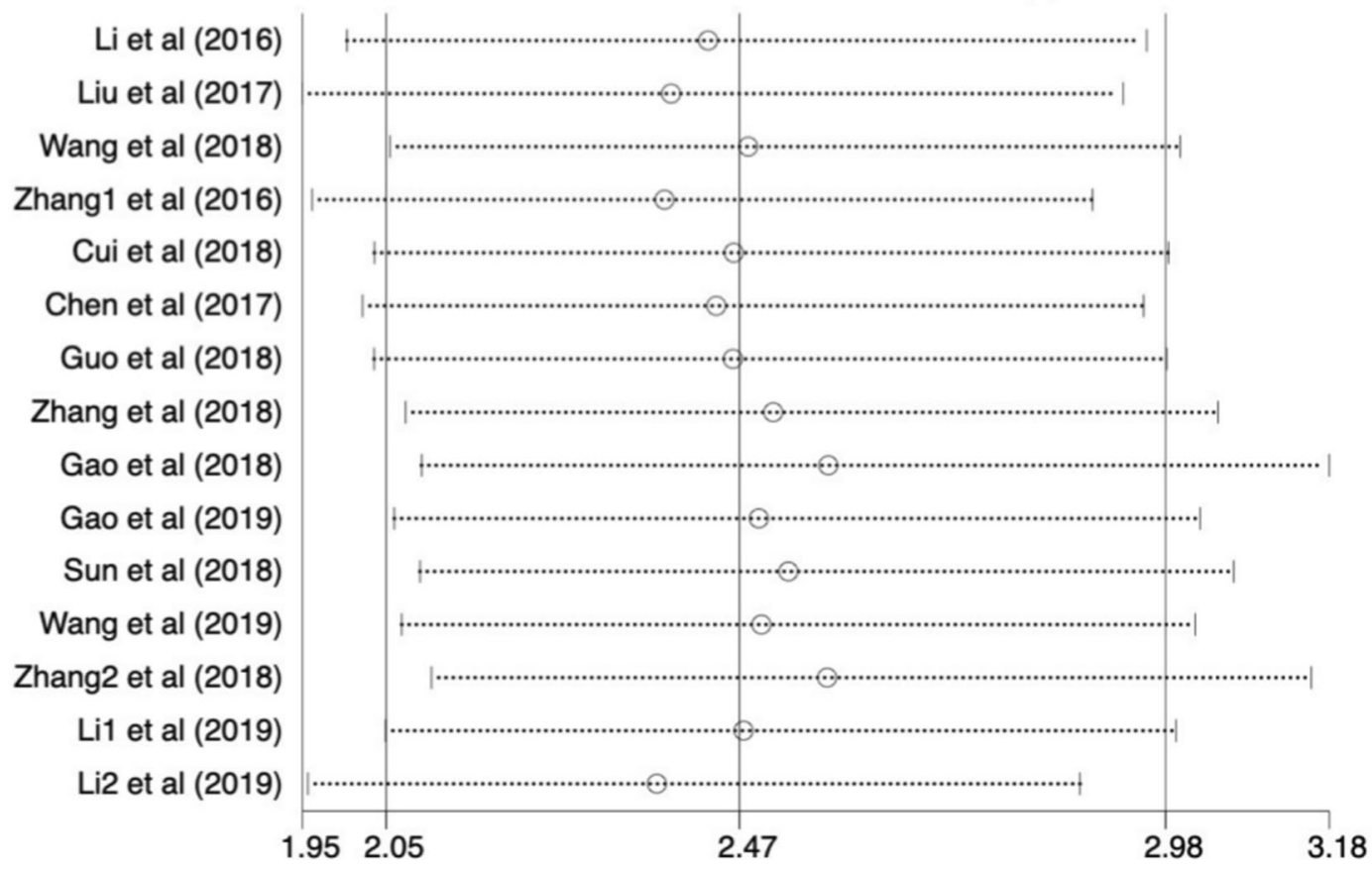

Fig. 5 Sensitivity analysis for the correlation between SNHG20 espression with overall survival (OS)

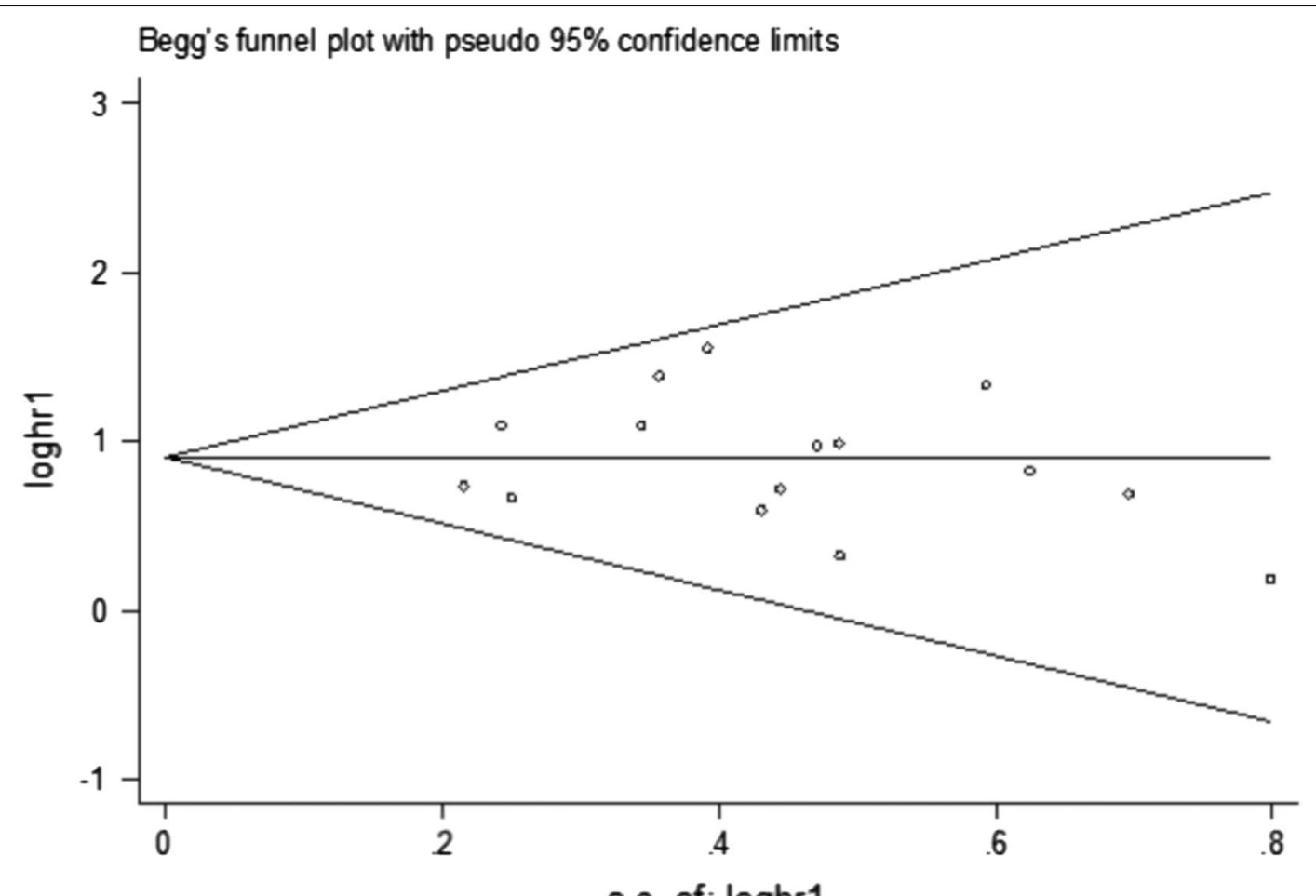

Fig. 6 Funnel plot of the publication bias for overall survival (OS) 
together with the outcomes of Begg's $(\mathrm{P}=0.553)$ and Egger's test $(\mathrm{P}=0.899)$, disclosed no distinct publication bias for OS. Furthermore, there was no evidence in favor of publication bias in terms of TNM stage, histological grade, and gender (Table 3). However, analysis of publication bias was inappropriate for tumor size, lymph node metastasis, tumor stage, smoking status, and distant metastasis, owing to the insufficient number of qualified publications in the meta-analysis.

\section{Discussion}

LncRNAs originally considered as transcriptional noise, have today been demonstrated to be implicated in manifold human malignancies [5]. Moreover, dysregulation of lncRNAs has been correlated with cancer cellular development by interfering with alternative splicing of pre-mRNA, by acting as a regulator in the transcription factor and histone-modifying enzyme, or by affecting the steps of translation and protein folding [26, 27]. Hence, the ectopic expression of lncRNAs could have a potential power for monitoring tumors and serving as a promising predictor of survival [28].

Previous published studies have elucidated that SNHG20 was a cancer-related lncRNA and had an indispensable role in oncogenic activity. For instance, SNHG20 has been demonstrated to promote tumor growth through functioning as a competing endogenous RNA (ceRNA) of miR-154 in non-small cell lung cancer and modulating the expression of ZEB2 and RUNX2 [29]. Additionally, SNHG20 could exert its carcinogenic action in breast cancer, and high level of SNHG20 could facilitate the proliferation, invasion and metastasis of cancer cells via modulating miR-495/HER2 axis [30]. Meanwhile, SNHG20 also elevated tumor progression by controlling the epithelial-to-mesenchymal transition (EMT) signaling pathway in osteosarcoma or hepatocellular carcinoma [23, 24, 31], activating PI3K/Akt/mTOR pathway in glioblastoma [25], as well as upregulating the expression of transforming growth factor- $\beta 1$ (TGF- $\beta 1$ ) in nasopharyngeal carcinoma [18], etc. Given these above molecular mechanisms of SNHG20 among various carcinomas, it was obvious that SNHG20 was connected with an unfavorable prognosis in cancer patients, which further provided support for clinical utility of SNHG20.

We aimed at exploring the relationship between SNHG20 expression levels and prognosis of human carcinomas in the present comprehensive meta-analysis, which pooled a total of 15 independent studies with 1187 tumor sufferers. The results of the research uncovered that enhanced SNHG20 expression was predominantly interrelated with short OS in cancer patients. Subgroup analyses were exploited to maximize clinical relevance. In the subgroup analysis according to cancer types, augmented SNHG20 expression was significantly related to poor OS in respiratory system cancers, gliomas, digestive system cancers, head and neck neoplasms as well as osteosarcomas, but not in cancers of the reproductive system. The reasons for the above phenomenon may be the difference in the age distribution of patients or the origin of tumor cells. Besides, we discovered that neither sample sizes nor data extraction methods altered the overall results. Subsequently, the outcomes from the sensitivity analysis and publication bias assessment also further verified the representativeness and reliability of our analysis. In addition, we identified that there was a strong link between SNHG20 overexpression and unfavorable DFS/RFS/PFS, meaning that cohorts with elevated SNHG20 expression exhibited a higher risk of tumor relapse or progression. Likewise, the clinicopathologic analyses manifested that patients with the high SNHG20 expression levels had increased occurrence probability of advanced TNM stage, large tumor size, positive lymph node metastasis, high tumor stage, and poorly differentiated grade. However, no prominent correlation was found between SNHG20 expression and gender, smoking status or distant metastasis. Taken together, the anomalous modulation of SNHG20 across different kinds of cancers suggests that SNHG20 is qualified as a candidate biomarker for both forecasting poor prognosis and providing therapeutic targets in cancer patients.

Nonetheless, we acknowledge several limitations in this work that should be pointed out. First of all, each and every enrolled study was performed in China, which increases the risk of geographical bias. Second, the sample sizes of some research and the included cancer types were comparatively smaller, which may bring about small-study effects. Third, the Cut-off level of high and low SNHG20 expression level was distinct across studies and not all of them provided this parameter, which perhaps reduces the reliability of our results. Fourth, HR with 95\% CI was indirectly reckoned by survival curves in some papers, which are less precise than those directly extracted from the original articles. Last, despite our primary outcomes were lack of heterogeneity, the predictive significance of SNHG20 in multiple human tumors might be exaggerated to some extent. Consequently, high-quality studies that are at large-scale are necessary for the verification of our conclusion.

\section{Conclusions}

In aggregate, the present meta-analysis elucidated that elevated SNHG20 expression is frequent in plenty of various types of cancers and qualified as a dependable and novel predictive factor of poor prognosis and clinicopathological features in cancer patients. Nevertheless, higher-quality multicenter studies with a larger sample 
capacity are still needed to corroborate and enhance the clinical application of SNHG20 in human malignancies.

\begin{abstract}
Abbreviations
SNHG20: Small nucleolar RNA host gene 20; LncRNAs: Long non-coding RNAs; ceRNA: Competing endogenous RNA; OS: Overall survival; DFS: Disease-free survival; RFS: Recurrence-free survival; PFS: Progression-free survival; qRT-PCR: Quantitative reverse transcription polymerase chain reaction; HCC: Hepatocellular carcinoma; ISH: In situ hybridization; TNM: Tumour-node-metastasis; TGF- $\beta 1$ : Transforming growth factor- $\beta 1$; NOS: Newcastle-Ottawa Scale; EMT: Epithelial-to-mesenchymal transition; HR: Hazard ratio; OR: Odds ratio; $\mathrm{Cl}$ : Confidence interval.
\end{abstract}

\section{Acknowledgements}

Not applicable.

\section{Authors' contributions}

HLZ, SZ, RNJ contributed to conception and design of the study. HLZ and QPL collected and analyzed the data. FW and XXG made some tables and figures. HLZ and SZ wrote the manuscript. HSW, RYT and XCW participated in the revision of the manuscript. All authors reviewed the final manuscript. All authors read and approved the final manuscript.

\section{Funding}

This research work was supported by the Project of Standard Diagnosis and Treatment of Key Disease of Jiangsu Province (BE2015722).

\section{Availability of data and materials}

The datasets during the current study are available from the corresponding author on reasonable request.

\section{Ethics approval and consent to participate}

Not applicable.

\section{Patient consent for publication}

Not applicable.

\section{Competing interests}

The authors declare that they have no competing interests.

Received: 23 November 2019 Accepted: 6 July 2020

Published online: 11 July 2020

\section{References}

1. Siegel RL, Miller KD, Jemal A. Cancer statistics, 2019. CA Cancer J Clin. 2019;69(1):7-34

2. Siegel RL, Miller KD, Jemal A. Cancer statistics, 2018. CA Cancer J Clin. 2018;68(1):7-30.

3. Huynh NP, Anderson BA, Guilak F, McAlinden A. Emerging roles for long noncoding RNAs in skeletal biology and disease. Connect Tissue Res. 2017;58(1):116-41.

4. Gugnoni M, Ciarrocchi A. Long noncoding RNA and epithelial mesenchymal transition in cancer. Int J Mol Sci. 2019;20(8):1924.

5. Miranda-Castro R, de-Los-Santos-Alvarez N, Lobo-Castanon MJ. Long noncoding RNAs: from genomic junk to rising stars in the early detection of cancer. Anal Bioanal Chem. 2019:411(19):4265-75.

6. Sanchez Calle A, Kawamura Y, Yamamoto Y, Takeshita F, Ochiya T. Emerging roles of long non-coding RNA in cancer. Cancer Sci. 2018;109(7):2093-100.

7. Zhao W, Ma X, Liu L, Chen Q, Liu Z, Zhang Z, Ma S, Wang Z, Li H, Wang Z, et al. SNHG20: a vital IncRNA in multiple human cancers. J Cell Physiol. 2019;11:3238-45.

8. Zhang D, Cao C, Liu L, Wu D. Up-regulation of LncRNA SNHG20 predicts poor prognosis in hepatocellular carcinoma. J Cancer. 2016;7(5):608-17.

9. Zhao Q, Gao S, Du Q, Liu Y. Long non-coding RNA SNHG20 promotes bladder cancer via activating the Wnt/beta-catenin signalling pathway. Int J Mol Med. 2018;42(5):2839-48.
10. Wang W, Luo P, Guo W, Shi Y, Xu D, Zheng H, Jia L. LncRNA SNHG20 knockdown suppresses the osteosarcoma tumorigenesis through the mitochondrial apoptosis pathway by miR-139/RUNX2 axis. Biochem Biophys Res Commun. 2018;503(3):1927-33.

11. Li XS, Shen FZ, Huang LY, Hui L, Liu RH, Ma YJ, Jin BZ. IncRNA small nucleolar RNA host gene 20 predicts poor prognosis in glioma and promotes cell proliferation by silencing P21. Onco Targets Ther. 2019:12:805-14.

12. Li C, Zhou L, He J, Fang XQ, Zhu SW, Xiong MM. Increased long noncoding RNA SNHG20 predicts poor prognosis in colorectal cancer. BMC Cancer. 2016;16:655.

13. Cui N, Liu J, Xia H, Xu D. LncRNA SNHG20 contributes to cell proliferation and invasion by upregulating ZFX expression sponging miR-495-3p in gastric cancer. J Cell Biochem. 2019;120(3):3114-23.

14. Chen Z, Chen X, Chen P, Yu S, Nie F, Lu B, Zhang T, Zhou Y, Chen Q, Wei C, et al. Long non-coding RNA SNHG20 promotes non-small cell lung cancer cell proliferation and migration by epigenetically silencing of P21 expression. Cell Death Dis. 2017;8(10):e3092

15. Guo H, Yang S, Li S, Yan M, Li L, Zhang H. LncRNA SNHG20 promotes cell proliferation and invasion via miR-140-5p-ADAM10 axis in cervical cancer. Biomed Pharmacother. 2018;102:749-57.

16. Zhang C, Jiang F, Su C, Xie P, Xu L. Upregulation of long noncoding RNA SNHG20 promotes cell growth and metastasis in esophageal squamous cell carcinoma via modulating ATM-JAK-PD-L1 pathway. J Cell Biochem. 2019;120:11642-50.

17. Gao P, Fan R, Ge T. SNHG20 serves as a predictor for prognosis and promotes cell growth in oral squamous cell carcinoma. Oncol Lett. 2019;17(1):951-7.

18. Sun C, Sun Y, Zhang E. Long non-coding RNA SNHG20 promotes nasopharyngeal carcinoma cell migration and invasion by upregulating TGFbeta1. Exp Ther Med. 2018;16(6):4967-74.

19. Wang D, Dai J, Hou S, Qian Y. LncRNA SNHG20 predicts a poor prognosis and promotes cell progression in epithelial ovarian cancer. Biosci Rep. 2019. https://doi.org/10.1042/BSR20182186.

20. Li Y, Xu J, Guo YN, Yang BB. LncRNA SNHG20 promotes the development of laryngeal squamous cell carcinoma by regulating miR-140. Eur Rev Med Pharmacol Sci. 2019;23(8):3401-9.

21. Tierney JF, Stewart LA, Ghersi D, Burdett S, Sydes MR. Practical methods for incorporating summary time-to-event data into meta-analysis. Trials. 2007;8:16.

22. Parmar MK, Torri $V$, Stewart L. Extracting summary statistics to perform meta-analyses of the published literature for survival endpoints. Stat Med. 1998;17(24):2815-34.

23. Liu J, Lu C, Xiao M, Jiang F, Qu L, Ni R. Long non-coding RNA SNHG20 predicts a poor prognosis for HCC and promotes cell invasion by regulating the epithelial-to-mesenchymal transition. Biomed Pharmacother. 2017:89:857-63.

24. Zhang J, Ju C, Zhang W, Xie L. LncRNA SNHG2O is associated with clinical progression and enhances cell migration and invasion in osteosarcoma. IUBMB Life. 2018;70(11):1115-21.

25. Gao XF, He HQ, Zhu XB, Xie SL, Cao Y. LncRNA SNHG20 promotes tumorigenesis and cancer stemness in glioblastoma via activating PI3K/Akt/mTOR signaling pathway. Neoplasma. 2019;66:532-42.

26. Sarfi M, Abbastabar M, Khalili E. Long noncoding RNAs biomarker-based cancer assessment. J Cell Physiol. 2019;234(10):16971-86.

27. Bonasio R, Shiekhattar R. Regulation of transcription by long noncoding RNAs. Annu Rev Genet. 2014;48:433-55.

28. Balas MM, Johnson AM. Exploring the mechanisms behind long noncoding RNAs and cancer. Noncoding RNA Res. 2018;3(3):108-17.

29. Lingling J, Xiangao J, Guiqing H, Jichan S, Feifei S, Haiyan Z. SNHG20 knockdown suppresses proliferation, migration and invasion, and promotes apoptosis in non-small cell lung cancer through acting as a miR-154 sponge. Biomed Pharmacother. 2019;112:108648.

30. Guan YX, Zhang MZ, Chen XZ, Zhang Q, Liu SZ, Zhang YL. Lnc RNA SNHG20 participated in proliferation, invasion, and migration of breast cancer cells via miR-495. J Cell Biochem. 2018;119(10):7971-81.

31. Pang A, Carbini M, Moreira AL, Maki RG. Carcinosarcomas and related cancers: tumors caught in the act of epithelial-mesenchymal transition. J Clin Oncol. 2018:36(2):210-6.

\section{Publisher's Note}

Springer Nature remains neutral with regard to jurisdictional claims in published maps and institutional affiliations. 